\title{
FUNDAMENTOS DA GESTÃO DO DESIGN E DA SUSTENTABILIDADE NO DESIGN DE MOBILIÁRIO
}

Raquel Brocco

Universidade Federal do Paraná

raquelbrocco@gmail.com

Liliane Iten Chaves

Universidade Federal Fluminense

chaves.liliane@gmail.com
Adriano Heemann

Universidade Federal do Paraná

adriano.heemann@gmail.com

Resumo: O presente artigo apresenta uma discussão a respeito das possíveis inter-relações teóricas que podem ser suscitadas entre os temas Gestão de Design, sustentabilidade e desenvolvimento de mobiliário. O procedimento adotado nesta investigação é a Revisão Bibliográfica Sistemática (RBS), realizada conforme o roteiro simplificado proposto por Conforto et al. (2011). Desse modo, o artigo apresenta o mapeamento e a discussão sobre as principais publicações brasileiras disponíveis nas bases Periódicos da Capes, Isi Web Of Knowledge e Science Direct sobre os temas enfocados no estudo. Finalmente, o artigo aponta para a existência de uma importante lacuna teórica na Gestão de Design e sugere possibilidades para pesquisas futuras neste campo.

Palavras-chave: Gestão de design, sustentabilidade, desenvolvimento de mobiliário.

\begin{abstract}
This article presents a discussion of possible theoretical interrelations that can be raised among the topics Design Management, sustainability and development of furniture. The procedure adopted in this research is the Bibliographical Systematic Review (RBS), performed according to the simplified script proposed by Comfort et al. (2011). Thus, the article presents the mapping and discussion of the main Brazilian publications available on the basis Journals Capes, Isi Web Of Knowledge and Science Direct on the topics focused on the study. Finally, the article points to the existence of an important theoretical gap in Design Management and suggests possibilities for future research in this field.
\end{abstract}

Key-words: design management, sustainability, furniture development. 


\section{INTRODUÇÃO}

A gestão do Design é cada vez mais considerada uma importante ferramenta de inovação pelas empresas, que pode diferencia-las e trazer vantagem competitiva. É amplamente reconhecido que essa ferramenta pode ser utilizada para influenciar, direcionar e integrar as ações de design dentro das organizações; treinar e auxiliar gerentes e parceiros na compreensão de design assim como designers na compreensão da gestão. (MOZOTA, 2011)

A inserção do design em todos os níveis da empresa une as visões e planos futuros dos órgãos decisores da organização aos projetos e produtos. Com isso, contribui na construção do valor estratégico da empresa, valor de marca e produto (FERREIRA, 2010). Conforme Martins e Merino (2011, p.26) "o campo de atuação do Design transcende a criação de produtos e peças gráficas como elementos isolados, passando a ser parte de um sistema e consolidando-se como um processo de gestão".

Contudo, com as consequências da globalização, da mudança do poder aquisitivo da sociedade e das novas políticas socioeconômicas, as empresas criadoras de bens e serviços são também frequentemente pressionadas a buscar por novas estratégias, reavaliando seus modelos de gestão atuais para modelos mais flexíveis, horizontais e sustentáveis. (MARTINS E MERINO, 2011)

Manzini e Vezzoli (2011), por sua vez, argumentam sobre a importância de práticas orientadas a sustentabilidade no contexto empresarial e questionam determinados modelos de desenvolvimento. Dentro deste contexto, o designer emerge como um importante agente no processo de transição para uma sociedade sustentável, ao apresentar os cenários factíveis e visões compartilhadas sobre futuros possíveis e sustentáveis para as organizações e aplicando estratégias ambientais conscientes desde as fases iniciais do projeto. (FERREIRA, 2010)

Assim, o presente estudo visa explorar e discutir inter-relações conceituais entre sustentabilidade e gestão de design no contexto específico do setor de mobiliário. Mais especificamente, a investigação é motivada pela seguinte pergunta de pesquisa: Quais as ênfases e lacunas existentes na integração dos temas da gestão do design com a sustentabilidade no desenvolvimento de mobiliário, nas publicações científicas atuais?

Portanto, o estudo aqui relatado tem como objetivo identificar as principais ênfases e lacunas de pesquisa sobre o assunto. Como método, uma RBS é descrita com o intuito de investigar os fundamentos para a pesquisa de mestrado que trata do tema de gestão de design e sustentabilidade no desenvolvimento de mobiliário. A revisão segue o roteiro proposto por Conforto et al. (2011), que conforme os autores, pode ser dividida em três fases principais: entrada, processamento e saída. Os resultados obtidos apontam uma importante carência de estudos publicados sobre gestão de design orientado a sustentabilidade no setor de mobiliário.

\section{DESENVOLVIMENTO}

Como método de levantamento de dados, utiliza-se uma RBS com objetivo de identificar e coletar as principais ênfases e lacunas de pesquisa na área de: gestão de design e sustentabilidade no desenvolvimento de mobiliário. A RBS é uma ferramenta que permite que o pesquisar desenvolva uma busca sistematizada sobre um tema específico. Por meio desse tipo de revisão, é possível mapear os trabalhos científicos já publicados 
com o intuito de desenvolver uma síntese do conhecimento, consolidar evidências, identificar lacunas e fornecer embasamento teórico. (CONFORTO et al, 2011)

As etapas da RBS realizada podem ser descritas em 15 etapas e divididas em 3 fases (CONFORTO et al, 2011), sendo elas: 1. Entrada: onde é definido o problema de pesquisa, os objetivos da RBS e as fontes primárias que auxiliam a definir as palavras-chave, definição das strings de busca e definição dos critérios de inclusão, qualificação, métodos, ferramentas e cronograma. 2. Processamento: é a fase na qual são realizadas as buscas, análise dos resultados da etapa de Entrada, documentação e arquivamento dos artigos selecionados. 3. Saída: é a fase final no qual são inseridos os "alertas" de busca, realizado - cadastro e arquivo dos artigos selecionados, a síntese dos resultados e possível construção de modelos teóricos. A Figura 1 ilustra as fases e etapas da RBS realizada neste estudo.

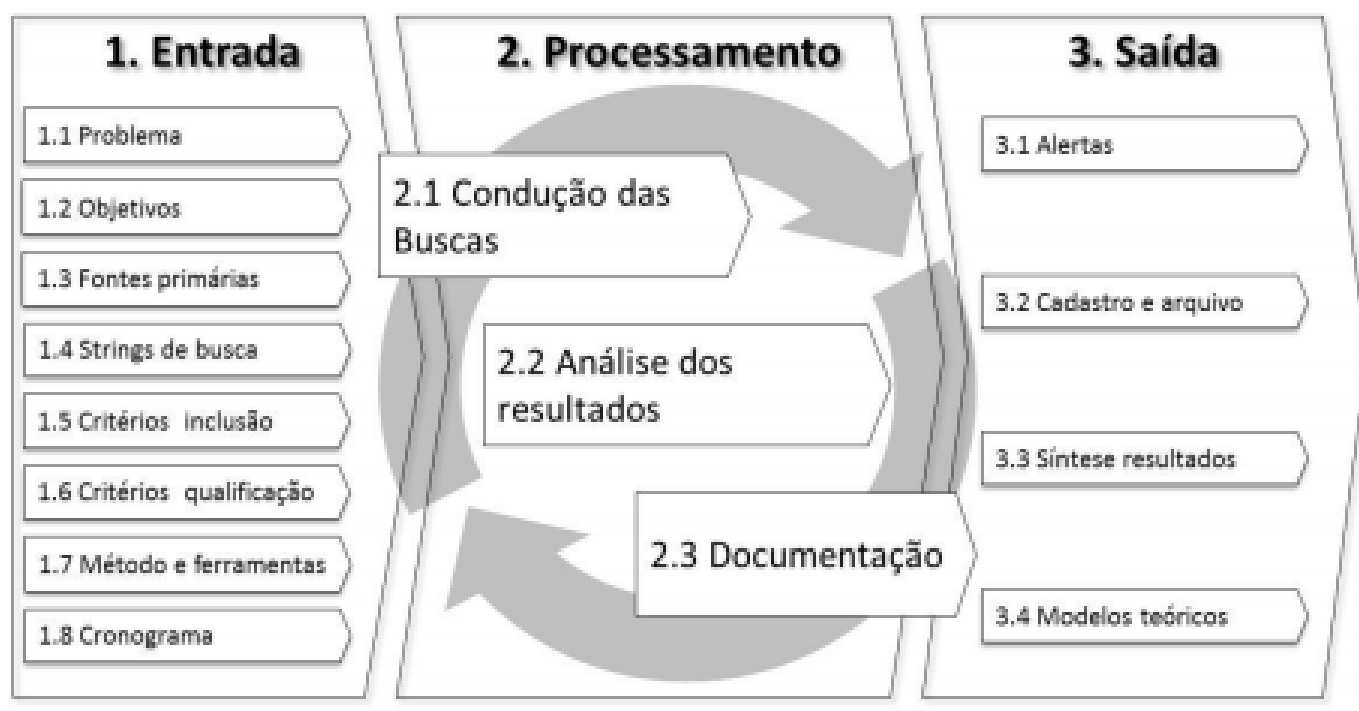

Figura 1. Modelo para condução da revisão bibliográfica Fonte: CONFORTO et al, 2011

A partir do estabelecimento do problema de pesquisa e do objetivo já mencionados na seção introdutória deste artigo, a fase de entrada utiliza inicialmente, como fontes primárias, a base de dados Google Acadêmico para definição preliminar das strings de busca para realização da RBS. Essa sondagem inicial no Google Acadêmico é estruturada da seguinte forma: 1. "Pesquisa na Web", com termo "gestão de design" em primeiro, associando-a as palavras "sustentabilidade" e "mobiliário". Essa busca resulta em 86 resultados. Uma segunda busca no Google Acadêmico utiliza os mesmos termos, porém na língua inglesa "design management", "sustainability" e "furniture", resultando em 1.410 indicações, que por sua vez são classificados por relevância. As mesmas palavraschaves são utilizadas para a formação das strings de busca, incluindo a combinação exclusiva dos termos "design management" e "sustainability".

Como critérios de inclusão, estão artigos que tratam do tema Gestão de Design em conformidade com a sustentabilidade, sendo este último termo inserido, ou não, no processo de desenvolvimento de mobiliário. Para os critérios de qualificação foi considerado (em todas bases de dados) o campo do design e a data de publicação. 
Para a busca nas bases de dados são utilizadas as strings de busca, seguindo a ordem: 1. "design management" AND "sustainability" AND "furniture"; 2. "design management" AND "sustainability".

\subsection{Resultados}

Os resultados da fase de processamento em função das strings utilizadas na fase de processamento são apresentados na Tabela 1:

Tabela 1- Resultados da RBS.

\begin{tabular}{|c|c|c|c|c|c|}
\hline \multirow[b]{2}{*}{ Strings de busca } & \multicolumn{5}{|c|}{ Base de dados } \\
\hline & $\begin{array}{l}\text { Periódicos } \\
\text { da Capes }\end{array}$ & $\begin{array}{l}\text { Isi Web Of } \\
\text { Knowledge }\end{array}$ & $\begin{array}{l}\text { Science } \\
\text { Direct }\end{array}$ & $\begin{array}{l}\text { Google } \\
\text { Acadêmico }\end{array}$ & \\
\hline $\begin{array}{l}\text { "design management" } \\
\text { AND "sustainability" AND } \\
\text { "furniture" }\end{array}$ & 3 & 1 & 0 & 1.220 & \\
\hline $\begin{array}{l}\text { "design management" } \\
\text { AND "sustainability" }\end{array}$ & 41 & 26 & 1 & 10.100 & \\
\hline Total encontrado & 44 & 27 & 1 & 11.320 & Total \\
\hline Total após filtros e seleção & 5 & 5 & 1 & 9 & 20 \\
\hline
\end{tabular}

Fonte: Elaborado pelo autor, com base na pesquisa realizada

Como pode ser observado, na base dos Periódicos da Capes a busca descreve a seguinte sequência:

1. "design management" AND "sustainability" AND "furniture", com três resultados.

2. "design management" AND "sustainability", com 41 resultados. Nenhum filtro específico foi aplicado. A seleção de cinco artigos relevantes do Periódicos da Capes se dá a partir da leitura dos título, resumo e palavras-chave.

Na base Isi Web Of Knowledge a busca é realizada com a seguinte sequência:

1. "design management" AND "sustainability AND "furniture", sendo obtido um resultado. 2. "design management" AND "sustainability", com filtro do ano 2005 até 2016, sendo obtidos 26 resultados. Após a leitura de título, resumo e palavras-chave cinco artigos são selecionados no Isi Web Of Knowledge.

$\mathrm{Na}$ base de dados Science Direct a busca realizada em "Title, Abstract, Keyword" descreve a seguinte sequência:

1. "design management" AND "sustainability", com filtro de 2005 até 2016, sendo obtidas 10 publicações. Após a leitura de título, resumo e palavras-chave um artigo é selecionado no Science Direct sequência:

A base de dados Google Acadêmico também é utilizada no processamento com a

1. "design management" AND "sustainability" AND "furniture", sendo encontrados 1.410 publicações. Com aplicação do filtro de 2005 até 2016 são obtidas 1.220 indicações.

2. "design management" AND "sustainability", com filtro de 2005 até 2016 resultando em 10.100 publicações. Os resultados foram apresentados por relevância pela base e após a leitura dos títulos a seleção resulta em nove artigos. 
Do final de 20 artigos obtidos por meio dos procedimentos descritos anteriormente, são selecionados nove trabalhos por meio da releitura de títulos, resumo e palavras-chave para leitura completa. Finalmente, quatro artigos se mostram diretamente relevantes para a resolução do problema de pesquisa.

Os quatro artigos foram sintetizados e apresentado seus resultados em um quadro (Quadro 1) apresentando os principais temas e conceitos abordados nas pesquisas, relacionado ao objetivo de estudo, salientando assim as ênfases e lacunas relacionadas ao tema de gestão de design e sustentabilidade no desenvolvimento de mobiliário.

Quadro 1- Síntese dos resultados da RBS.

\begin{tabular}{|l|l|l|l|l|}
\hline Temas e conceitos / autores & $\begin{array}{l}\text { Castro e } \\
\text { Cardoso } \\
(2010)\end{array}$ & $\begin{array}{l}\text { Ferreira } \\
(2010)\end{array}$ & $\begin{array}{l}\text { Fassi Jr } \\
(2014)\end{array}$ & $\begin{array}{l}\text { Fargnoli } \\
\text { et al. } \\
(2014)\end{array}$ \\
\hline $\begin{array}{l}\text { Importância da sustentabilidade nos } \\
\text { novos paradigmas econômicos }\end{array}$ & 2 & 1 & 1 & 2 \\
\hline Design e sustentabilidade & 2 & 1 & 1 & 2 \\
\hline $\begin{array}{l}\text { Estratégias de design para a } \\
\text { sustentabilidade }\end{array}$ & 2 & 1 & 1 & 2 \\
\hline $\begin{array}{l}\text { Gestão de design e sustentabilidade } \\
\text { Desenvolvimento de mobiliário e }\end{array}$ & 3 & 1 & 3 & 1 \\
\hline $\begin{array}{l}\text { Legenda: 1- Aborda / 2- aborda } \\
\text { parcialmente / 3- Não aborda }\end{array}$ & & 3 & 1 & 3 \\
\hline
\end{tabular}

Fonte: Elaborado pelo autor, com base na pesquisa realizada.

\subsection{Discussão}

Nesta seção é apresentada a compilação dos temas buscados pelos autores encontrados.

Conforme Fassi Jr (2014) e Ferreira (2010), com a globalização e avanço tecnológico a humanidade enfrenta uma nova relação referente as ações de desenvolver e conservar. A degradação ambiental desenfreada iniciada com a revolução industrial colocou em destaque as relações entre o consumo desenfreado, o crescimento demográfico e o uso de recursos ambientais. Dessa constatação emerge a importância de "avaliar a capacidade ecológica necessária para sustentar o consumo de produtos e estilos de vida" (FASSI JR, 2014 p. 1). Ainda, o processo de globalização acelerou também o aumento da pobreza mundial, desigualdade social e todas a suas consequências (FASSI JR, 2014).

De acordo com a literatura enfocada, para que o desenvolvimento sustentável seja buscado, é necessário a convergência de três forças: a sustentabilidade, o desenvolvimento e a produção industrial, todos em equilíbrio no âmbito ambiental, social e econômico (FASSI JR, 2014). De um modo geral, o desenvolvimento sustentável busca satisfazer as necessidades do presente sem comprometer que as gerações futuras possam satisfazer suas próprias necessidades, além de respeitar as diversidades culturais e potencializar as características regionais (FASSI JR, 2014; FERREIRA, 2010). Contudo, a esse respeito, cabe a reflexão crítica de que, como observado em escala global, tampouco as 
necessidades da sociedade presente são satisfeitas integralmente, o que levanta uma dúvida importante sobre a viabilidade de as gerações futuras alcançarem suas próprias satisfações.

Apesar dessa situação conceitual, o atual modelo produção, consumo e crescimento econômico parece estimular o consumo em demasia e influenciar "as diretrizes que norteiam os investimentos em design, inovação e marketing (uso intensivo da mídia falada e escrita) tem como objetivo principal o aumento de vendas de produtos, ou estímulo ao consumo, baseado, muitas vezes naquilo que não nos serve" (FASSI JR, 2014 p. 3).

De forma geral, as publicações de Cardoso (2010); Ferreira (2010); Fassi Jr (2014) e Fargnoli et al. (2014) estudadas apresentam duas responsabilidades do design no desenvolvimento sustentável. Primeiro, como participante ativo do desenvolvimento de novos produtos ou serviços, o design deve estar em conformidade com o desenvolvimento sustentável, não apenas internamente, na organização, mas também no contexto social em que a empresa é inserida. Segundo, baseado nas qualidades e obrigações do designer como influenciador cultural e difusor no estabelecimento de uma ética de consumo, enquanto solução para problemas ambientais e sociais. Fargnoli et al. (2014) também conceitua o termo Design para a Sustentabilidade. Apesar da sua conotação ecológica, há ainda a necessidade de uma abordagem mais holística centrada nas pessoas.

Dentro deste contexto, Ferreira (2010) e Fassi Jr (2014) acrescentam a importância da adoção de determinadas estratégias projetuais para o desenvolvimento sustentável, tais como: reduzir o uso de matéria e energia; escolher materiais e recursos de baixo impacto ambiental; estender a vida dos materiais (reciclagem ou reaplicação de materiais descartados); facilitar a desmontagem e otimizar a vida dos produtos não só em termos físicos, mas também em termos de aceitação do produto pelo mercado.

Como exemplo desta aplicação Fassi Jr (2014) apresenta iniciativas institucionais e profissionais de estabelecer uma ligação entre design e a questão ambiental que vêm acontecendo no Brasil nos últimos anos. Tal iniciativa tem motivação na preocupação com a retirada ilegal e sem controle de madeira da Floresta Amazônica e com o desperdício que ocorre neste setor. De acordo com o autor, o IBAMA tem trabalhado em um modelo para incentivar o uso em móveis de espécies manejadas de madeiras da floresta tropical brasileira. A instituição desenvolve parcerias com instituições de ensino de design e investe em exposições itinerantes intituladas "Design e Natureza", além de estimular o uso de madeiras brasileiras em concursos nacionais de design. O autor ressalta, contudo, que a maior parcela de designers do mercado moveleiro do sul do Brasil não compreende o significado do termo sustentabilidade, atrelando a ele conceitos confusos e superficiais. De acordo com pesquisa realizada, os designers de móveis em geral se sentem mais pressionados por outras imposições de mercado (como a de seguir tendências estilísticas ou a de reduzir custos) (FASSI JR, 2014).

Castro e Cardoso (2010) apresentam uma abordagem do design orientada a sustentabilidade, que tem se tornado conhecida também como um tipo de design estratégico. Conforme os autores, "o seu objetivo é a identificação de estratégias para inovações sistêmicas, inclusive novos produtos, serviços e sistemas que representem uma vantagem competitiva a longo prazo, e, neste caso, considera-se como objeto de estudo o sistema produto-serviço" (CASTRO E CARDOSO, 2010 p. 73 apud KRUCKEN, 2005).

Na possível inter-relação entre Gestão do Design e sustentabilidade, foco do presente estudo, Ferreira (2010) e Fargnolli et al. (2014) apontam a gestão como articuladora e influenciadora das atividades de design dentro das organizações. A gestão e fornece 
ferramentas e métodos para apoiar gestores e técnicos em todo o processo de design e atua como conector das decisões e objetivos estratégicos da empresa com o portfólio e projetos de novos produtos. A Gestão de Design também auxilia as organizações a criar cenários futuros em conformidade com os novos paradigmas sociais, ambientais e econômicos e, assim, pode melhorar seu grau de sustentabilidade.

\section{CONCLUSÃO}

O presente estudo aponta para a pertinência da inter-relação entre conceitos de Gestão de Design e sustentabilidade. Conforme as publicações consultadas, o design tem um papel de influência na performance de sustentabilidade de uma empresa e seus respectivos produtos ou serviços.

No âmbito da discussão apresentada, destacou-se a ambiguidade no entendimento do termo "sustentabilidade" no contexto da gestão. O presente artigo conclui que já existe um entendimento pontual de que a "Gestão Sustentável do Design" (FERREIRA, 2010) tenha como objetivo geral definir os pilares estruturantes do design para auxiliar as organizações na transição para um "novo paradigma".

A investigação também menciona publicações que apontam para o papel importante do designer como influenciador e articulador das mais variadas práticas nas organizações, o que sugere, de modo implícito, para a inclusão da sustentabilidade.

Das publicações detectadas por meio da RBS, apenas uma menciona a pertinência da sustentabilidade no design de mobiliário, embora aquela publicação também indique um relativo desconhecimento por parte de designers de móveis a respeito do assunto. $\mathrm{O}$ estudo não detectou publicações que triangulem os conceitos de gestão de design, sustentabilidade e design de móveis, o que constitui uma importante lacuna na teoria sobre o assunto.

Diante do exposto, recomenda-se que pesquisas futuras investiguem e elucidem tal triangulação a fim de contribuírem para o estabelecimento de estratégias orientadas a sustentabilidade em diferentes níveis da gestão de design de móveis. Nesse sentido, aponta-se como promissora a eventual construção de um termo próprio para uma abordagem desse tipo, que afaste possíveis confusões conceituais e que leve em consideração o papel do profissional de design no contexto mundial.

\section{AGRADECIMENTO}

Os autores agradecem a Fundação Araucária pelo apoio na forma de bolsa de estudos.

\section{REFERÊNCIAS}

CASTRO, M. L.; CARDOSO, J. ESTRATÉGIA E DESIGN: CONSTRUÇÃO DAS ABORDAGENS CONTEMPORÂNEAS. Strategic Design Research Journal, V. 3, N. 3, P. 69-75, 2010. DisPonível em: <HTTP://REVISTAS.UNISINOS.BR/INDEX.PHP/SDRJ/ARTICLE/VIEW/4789>. ACESSADO EM 17/03/2016

CONFORTO, E. C.; AMARAL, D. C.; SILVA, S. L. DA. ROTEIRO PARA REVISÃo BIBLIOGRÁfiCA SISTEMÁTICA: APLICAÇÃO NO DESENVOLVIMENTO DE PRODUTOS E GERENCIAMENTO DE PROJETOS. $8^{\circ}$ Congresso Brasileiro de GestÃo de DesenVolviemnto de Produto - CNGDP 2011, , N. 1998, P. 112, 2011. DISPONÍVEL EM: <HTTP://WWW.UFRGS.BR/CBGDP2011/DOWNLOADS/9149.PDF>. ACESSADO EM 17/03/2016

FARGNOLI, M.; DE MINICIS, M.; TRONCl, M. Design MANAGEMENT for SustaINABILITY: AN INTEGRATED APPROACH FOR THE DEVELOPMENT OF SUSTAINABLE PRODUCTS. JOURNAL OF ENGINEERING AND 
Technology Management - Jet-M, V. 34, P. 29-45, 2014. ElseVier B.V. Disponível em: <HTTP://DX.DOI.ORG/10.1016/J.JENGTECMAN.2013.09.005>. ACESSADO EM 17/03/2016

FASSI, E.; JR, C. InOVação TeCnológICA e Sustentabilidade : Possíveis ferramentas para UMA NECESSÁRIA INFERFACE. ReVISTA EdUCAÇÃo \& TECNOLOGIA, CURITIBA, V.8, P. 97-109, 2014. DISPONÍVEL EM:HTTP://WWW.UTFPR.EDU.BR/CURITIBA/ESTRUTURA-

UNIVERSITARIA/DIRETORIAS/DIRPPG/GRUPOS/TEMA/66INO_TEC_SUS_FERR_INTERF.PDF. ACESSADO EM $17 / 03 / 2016$

FERREIRA, M. M. GESTÃo do DESIGn E suA ADEQUAÇÃo A UM NOVO PARADIGMA REGIDO PELA Sustentabilidade. Faculdade de ARquitectura, Universidade TÉCNICA DE LisboA, P. 219, 2010. DISPONIVEL EM: HTTP://WWW.REPOSITORY.UTL.PT/HANDLE/10400.5/2913. ACESSADO EM: $17 / 03 / 2016$

MANZINI, Ezio; VeZzoli, Carlo; O Desenvolvimento de Produtos Sustentáveis. tradução de Astrid de Carvalho. 1ed. SÃo Paulo: Ed. Universidade de SÃo Paulo, 2005.

MARTINS, ROSANE FONSECA DE F. ; MERINO, E. A. D. A Gestão do Design COMO EstratéGIa ORGANIZACIONAL. 2. ED ED. LONDRINA: RIO BOOKS, 2011.

MOZOTA, B. B. DE. GESTÃo do DESIGN: USANDO O DESIGN PARA CONSTRUIR VALOR DE MARCA E InOVAÇÃo Corporativa. Porto Alegre, 2011. 